\title{
Research on the Dynamic Comprehensive Evaluation of Power Quality Based on the Improved Scatter Degree
}

\author{
Min GUO ${ }^{a}$, Weidong $\mathrm{CHEN}^{\mathrm{b}}$, Qingren $\mathrm{JIN}^{\mathrm{c}}$, Hongbo $\mathrm{CHU}^{\mathrm{d}}$ \\ Guangxi Power Grid Electric Power Research Institute, Nanning, 530023, China \\ aemail: guo_m.sy@gx.csg.cn, ${ }^{b}$ email:chen_wd.sy@gx.csg.cn \\ cemail: jin_qr.sy@gx.csg.cn, ${ }^{\mathrm{d}}$ email:chu_hb.sy@gx.csg.cn
}

\begin{abstract}
Keywords: Power Quality; Scatter Degree; $\mathrm{G}_{1}$ method; Grey Relational Analysis; Dynamic Comprehensive Evaluation
\end{abstract}

\begin{abstract}
In view of the sensitivity of power quality indices on different loads, a method for the dynamic comprehensive evaluation of power quality based on the improved scatter degree was put forward. Firstly, according to the sensitivity of power quality indices on different loads, the order relation among such indices is determine, the subjective weight vectors are obtained by the application of its analysis method, and the measured values of power quality indices are weighted according to those vectors, those subjective weighting values are turned into the objective weights by the scatter degree method, at this moment the objective weights are the comprehensive weight of the sensitivity of different power quality indices on the loads. Secondly the dynamic weight vectors are obtained by adjusting the comprehensive weights according to the distribution situation of the measured index values, considering the influence of the cask effect. Finally the level of the power quality at each monitoring point will be obtained by means of the dynamic weight vectors and grey relational analysis. The power quality evaluation program is based on the MATLAB software, analyses the measured values of $220 \mathrm{kV}$ Xindu substation and $110 \mathrm{kV}$ Huifeng substation in Guangxi, the results are proved that the improved method is reasonable and practical, compared with the results of the other methods in the references.
\end{abstract}

\section{Introduction}

At present the power industry faces the market, and introduces the competition mechanism, in order to make the cost minimum and the profit maximum, the level of power quality has become an important symbol of power system operation and management level [1]. The reasonable, fair and comprehensive evaluation of power quality is one of the prerequisites for establishing a fair electricity market, which prices in accordance with the quality of the power and makes clear the responsibility between the supply side and the demand side.

The papers [2-5] adopt the evaluation method based on the fuzzy theory, but the membership functions of that theories are determined in accordance with the experience or the statistics, thus the results are greatly influenced by the subjective factors. The papers [5] adopt the entropy method, which can modify the subjective weight by means of analytic hierarchy process (AHP), but the principle of the entropy method [12] is that the greater the difference among the measured values of the index for all the evaluated objects, the lager the weight of the index, vice versa. But the most important index may not make the attribute of all the evaluated objects with the largest difference, and the unimportant one may make the attribute of all the evaluated ones with the largest difference, therefore it may appear that the weight coefficient of the important index is small and the weight coefficient of the unimportant one is big. The Scatter degree (SD) method proposed in this paper highlights the differences among the evaluated objects from the whole, and overcomes the shortcoming of the entropy method. The papers [6-7] adopt the evaluation method based on the neural network are put forward. In the model the neural network method without the artificial weights can objectively reflect the relationship among the evaluation indices through the self-learning process of the neural network, but it needs a large number of the samples for training, 
and is easy to fall into the local optimum. In order to avoid falling into the local optimum, the paper [6] adopts the genetic algorithm that the initial values are optimized, and the evaluation requires a long time. The paper [7] adopts the neural network based on the radial basis function for the evaluation model, its calculation speed is faster than the BP model. The AHP method [8] obtains the subjective weight, but needs a large amount of the calculation and the consistency test, the order relation analysis method ( $G_{1}$ method) adopts in this paper can obtain the subjective weight, which is the improvement of the AHP method and can be made up for these shortcomings of the AHP method. The paper [9] adopts a method based on looking for traces of the genetic projection. Firstly projection indices through the random values are established within the scope of all levels, then the beset projection value of the samples is obtained from the best projection direction of those index samples according to the genetic algorithm, finally the scatter diagram between the best projection value and the level value can obtain the level of each evaluation object. But the selected projection index samples in the paper are only for all indices at the same level, and practically many indices are in different levels. Thus this method cannot make good evaluation when there is great difference for many indices at different levels. This is a very good solution to this problem by dynamically adjusting the comprehensive weight in this paper. In addition these existing evaluation methods do not consider the sensitivity of different power quality indices on different loads.

In view of the existing problems of those power quality comprehensive evaluation methods, this paper proposes a dynamic evaluation method considering load's sensitivity. Firstly the comprehensive weight is obtained from the improved SD based the $\mathrm{G}_{1}$ method, then a group of dynamic weights is obtained by adjusting the comprehensive ones according to the actual measured values of each bus. Finally the level of the power quality at each bus will be obtained by means of the grey relational analysis (GRA).

\section{Determination of the index weight}

The comprehensive evaluation of power quality is a problem of multi-index evaluation, the determination of index weight has a great influence on the rationality of the evaluation conclusion. At present, the method of determining the weight can be divided into the subjective weighting method and the objective one [10]. The subjective weighting method can reflect the decision-maker's will, but will be affected by the decision-maker's understanding of the scope. The method to determine the index weight depends on its own meaning of power quality, the method in the paper [11] called the meaning weight. The objective weighting method can be on the basis of strong mathematical theory, and avoids the influence of the artificial factors, but cannot reflect the decision-maker's will. The determination of the weight can be relevant to its data structure and trend, the method in the paper [11] called the structural weight of the data. Two methods have their advantages and disadvantages, so it is necessary to adopt the combination of the subjective method and the objective one for determination the weight.

\section{Subjective weight determinated by the $G_{1}$ method}

The $\mathrm{G}_{1}$ method is a new method which does not need to check the consistency of the subjective weighting [12-13].

The steps of determining the subjective weight with the $G_{1}$ method:

1) The order relation among all power quality indices is determinated by the sensitivity of different power quality indices on the basis of the load, as shown in the formula (1).

Definition: if the importance of the evaluation index $x_{i}$ with respect to an evaluation criteria (or target) is greater than (or not less than) the evaluation index $x_{j}$, it is denoted as $x_{i} \succ x_{j}$.

The order relation of the index $x_{1}, x_{2}, \cdots x_{m}$ is determinated with the symbol " $\succ$ ", as shown below:

$$
x_{1}^{*} \succ x_{2}^{*} \succ \cdots \succ x_{m}^{*}
$$


In the type: $x_{i}^{*}$ stands for the $i$ index of the evaluation after sorting $\left\{x_{i}\right\}$ as the order relation “ $\succ, i=(1,2, \cdots, m)$. For the convenience of writing, the following of $x_{i}^{*}$ is replaced for $x_{i}$. The specific method for determining on the formula (1) of the order relation refers to the paper [12].

2) The variable $r_{k}$ is the comparison and judgment of the relative importance between $x_{k}$ and $x_{k+1}$, its value refers to table 1 , as shown in the formula (2).

$$
r_{k}=x_{k} / x_{k}(k=m-1, \cdots, 3,2,1)
$$

Table 1 reference value of the variable $r_{k}$

\begin{tabular}{cc}
\hline$r_{k}$ & Remark \\
\hline 1.0 & The index $x_{k}$ is the same importance as the index $x_{k+1}$ \\
1.2 & The index $x_{k}$ is a little more importance than the index $x_{k+1}$ \\
1.4 & The index $x_{k}$ is significantly more importance than the index $x_{k+1}$ \\
1.6 & The index $x_{k}$ is strongly more importance than the index $x_{k+1}$ \\
1.8 & The index $x_{k}$ is extremely more importance than the index $x_{k+1}$ \\
\hline
\end{tabular}

3) The weight coefficient $\omega_{k}$ is calculated according to the formula (3) and (4).

$$
\begin{aligned}
& \omega_{m}=\left(1+\sum_{k=1}^{m-1} \prod_{i=k}^{m-1} r_{i}\right)^{-1} \\
& r_{k}=\omega_{k} / \omega_{k+1}(k=m-1, \cdots, 3,2,1)
\end{aligned}
$$

\section{Objective weight determinated by the SD method}

The evaluated objects of $n$ and the evaluated indices of $m$ compose of the matrix $n \times m$ by the SD method. The weight coefficient of each index makes the difference of each evaluated object as great as possible. The $m$-dimension evaluation space composes of the best one-dimension space, makes the projection of each vector scattered in one-dimension space, called the maximum degree of the scatter [14].

Supposed the matrix of the measured indices according to the formula (5).

$$
\mathbf{X}=\left[\begin{array}{cccc}
x_{11} & x_{12} & \cdots & x_{1 m} \\
x_{21} & x_{22} & \cdots & x_{2 m} \\
\vdots & \vdots & & \vdots \\
x_{n 1} & x_{n 2} & \cdots & x_{n m}
\end{array}\right]
$$

In the type: $n$ stands for the amount of the evaluated objects, $m$ stands for the amount of the measured indices.

Normalized the matrix $\mathbf{x}$ according to the formula (6).

$$
x_{i j}{ }^{*}=\frac{x_{i j}-\overline{x_{j}}}{s_{j}}
$$

In the type: $\overline{x_{j}} 、 s_{j}(j=1,2, \ldots, m)$ respectively stands for the mean and the variance of the measured index $j$. For the convenience of writing, the following of $x_{i j}^{*}$ is replaced for $x_{i j}$.

The principle of the weight coefficient by the SD method could reflect the difference among all evaluated objects which is of different "quality" the best. That makes the variable $y_{i}$ of the formula (7) as maximum as possible.

$$
y_{i}=\omega_{1} x_{i 1}+\omega_{2} x_{i 2}+\cdots+\omega_{m} x_{i m}(i=1,2, \cdots n)
$$

Formula (7) is written the form of the matrix, as shown below:

$\mathbf{y}=\mathbf{X} \boldsymbol{\omega}$

In the type: $\mathbf{y}=\left[y_{1}, y_{2}, \cdots, y_{n}\right]^{T}$.

The projection points of $n$ form the sample $\left\{y_{i}\right\}(i=1,2, \cdots, n)$, its variance as shown below:

$$
s^{2}=\frac{1}{n} \sum_{i=1}^{n}\left(y_{i}-\bar{y}\right)^{2}=\frac{\mathbf{y}^{\mathbf{T}} \mathbf{y}}{n}-\bar{y}^{2}
$$


By normalizing the original data, $\bar{y}=0$ is made, and the equation (10) forms by the formula (8) and (9).

$$
n s^{2}=\omega^{\mathrm{T}} \mathbf{X}^{\mathrm{T}} \mathbf{X} \boldsymbol{\omega}=\boldsymbol{\omega}^{\mathrm{T}} \mathbf{H} \boldsymbol{\omega}
$$

In the type: $\mathbf{H}=\mathbf{X}^{\mathrm{T}} \mathbf{X}$ is a symmetric matrix.

Limited condition $\omega^{\mathbf{T}} \boldsymbol{\omega}=1$, the maximum of the formula (10) is to be solved, it can meet the condition of the formula (11).

$$
\left\{\begin{array}{c}
\max \boldsymbol{\omega}^{\mathrm{T}} \mathbf{H} \boldsymbol{\omega} \\
\text { s.t. } \boldsymbol{\omega}^{\mathrm{T}} \boldsymbol{\omega}=1 \\
\omega>0
\end{array}\right.
$$

When $\boldsymbol{\omega}$ is the characteristic vector corresponding to the maximum value of $\mathbf{H}$, the formula (10) can reach its maximum value [13]. Hence, the characteristic vector $\boldsymbol{\omega}$ corresponds to the maximum characteristic value of $\mathbf{H}$, and the weight coefficient vector $\boldsymbol{\omega}=\left[\omega_{1}, \omega_{2}, \cdots, \omega_{m}\right]^{T}$ after normalization, furthermore

$$
\sum_{j=1}^{m} \omega_{j}=1
$$

The SD method emphasizes that the difference among the evaluated objects will be taken overall, its premise is the same importance of each index which is relative to the purpose of the evaluation. In fact, the importance of each index is not equal in general. Therefore, the SD method must be improved [12].

The steps for the improvement:

1) The subjective weight coefficient $p_{j}(j=1,2, \ldots, m)$ of all power quality indices by the $G_{1}$ method, on the basis, the measured values of the index need to weight process, that is, Supposed:

$x_{i j}{ }^{*}=p_{j} x_{i j}(j=1,2, \cdots, m ; i=1,2, \cdots, n)$

2) The objective weight can be obtained after the application of the SD method deals with those weighted data, that is, the comprehensive weight $w_{j}(j=1,2, \ldots, m)$.

\section{Dynamic adjustment of the weight}

Power quality is affected by many factors, if certain index worsens sharply, the overall power quality will be decreased [4].

The principle of the cask effect [15], think the maximum interference index often determines the operation state of the equipment, the level of the maximum interference index is the one of the comprehensive evaluation in power quality.

In this paper the weight coefficient of the index can be dynamically adjusted according to the cask effect. For the "very small” index, the greater its measured values, the greater its severity, so its weight should be increased. The weight of the index should be corrected according to the severity of the index.

The correction process is as follow:

1) The severity of the index is calculated as formula (14):

$\omega_{i j}{ }^{\prime}=\frac{x_{i j}{ }^{\prime \alpha}}{\sum_{j=1}^{m} x_{i j}{ }^{\prime \alpha}}$

In the type: $x_{i j}{ }^{\prime}=\frac{x_{i j}}{x_{j \lim }}, x_{j \lim }$ is the standard value of the index $\mathrm{j} ; \alpha$ is the adjustment coefficient, and its value is set to 1 in this paper.

2) Those values which are obtained from the formula (14), the comprehensive weight $w_{j}$ is dynamically adjusted by the formula (15).

$\omega_{i j}{ }^{\prime \prime}=\omega_{i j}{ }^{\prime} \cdot \omega_{j}$

3) The adjusted weight is normalized by the formula (16), and then the dynamic weight is 
obtained.

$$
\omega_{i j}=\frac{\omega_{i j}{ }^{\prime \prime}}{\sum_{j=1}^{m} \omega_{i j}^{\prime \prime}}
$$

\section{Selection of the index and classification of each index in power quality}

All the countries in the world specify the corresponding power quality standards according to the actual situation. So far, China has specified and issued six national standards of power quality. Specific: the limit value of each index is averagely divided into 5 levels, for the classification of the qualified; the value beyond the limit is divided into 4 levels, and its span is 2 times the one of the qualified. From the 1 9 level, the situation of power quality is down step by step, as shown in figure 1.

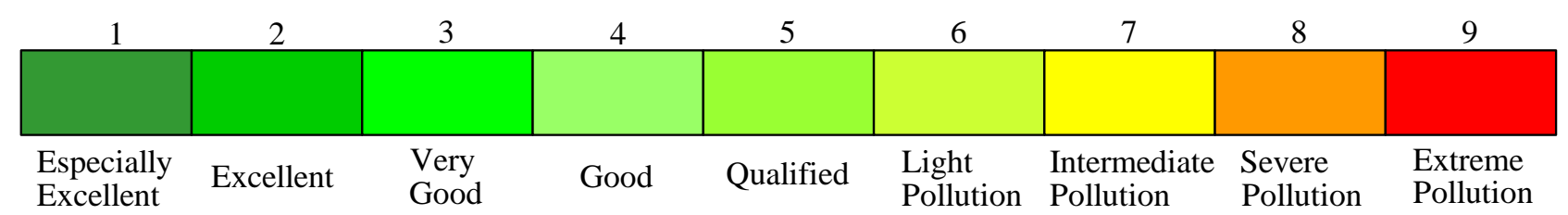

Figure 1 the classification of the power quality

This paper selects the indices and classifies the levels of power quality [9], as shown in table 2.

Table 2 the index selection and classification standard $(110 \mathrm{kV})$

\begin{tabular}{|c|c|c|c|c|c|}
\hline Level Index & $\begin{array}{c}\text { Voltage Deviation } \\
x_{1} \%\end{array}$ & $\begin{array}{c}\text { Frequency } \\
\text { Deviation } \quad x_{2}\end{array}$ & $\begin{array}{l}\text { Total Harmonic } \\
\text { Distortion } \quad x_{3} \%\end{array}$ & $\begin{array}{l}\text { Long Time } \\
\text { Flicker } \quad x_{4}\end{array}$ & $\begin{array}{c}\text { Three Phase Unbalance } \\
\text { Degree } X_{5} \%\end{array}$ \\
\hline 1 & $\leq 2$ & $\leq 0.04$ & $\leq 0.4$ & $\leq 0.16$ & $\leq 0.4$ \\
\hline 2 & $\leq 4$ & $\leq 0.08$ & $\leq 0.8$ & $\leq 0.32$ & $\leq 0.8$ \\
\hline 3 & $\leq 6$ & $\leq 0.12$ & $\leq 1.2$ & $\leq 0.48$ & $\leq 1.2$ \\
\hline 4 & $\leq 8$ & $\leq 0.16$ & $\leq 1.6$ & $\leq 0.64$ & $\leq 1.6$ \\
\hline 5 & $\leq 10$ & $\leq 0.2$ & $\leq 2$ & $\leq 0.8$ & $\leq 2$ \\
\hline 6 & $\leq 14$ & $\leq 0.28$ & $\leq 2.8$ & $\leq 1.12$ & $\leq 2.8$ \\
\hline 7 & $\leq 18$ & $\leq 0.36$ & $\leq 3.6$ & $\leq 1.44$ & $\leq 3.6$ \\
\hline 8 & $\leq 22$ & $\leq 0.44$ & $\leq 4.4$ & $\leq 1.76$ & $\leq 4.4$ \\
\hline 9 & $>22$ & $>0.44$ & $>4.4$ & $>1.76$ & $>4.4$ \\
\hline
\end{tabular}

\section{Comprehensive evaluation of the level using GRA method in power quality}

On the basis of various factors' samples, GRA uses grey relational degree to describe the strength, the size and the order of the relationship among factors [14]. If the samples show that changes between two factors(size direction、 speed and so on) are basically the same, then the relation between them is larger, and vice versa. Therefore, the ideal index value of power quality would be a sample for the reference, with the index of comparative samples worsens, the relation between the comparative sample and the reference one could be smaller, and then its level will be bigger. The boundary of the correlation classification can be obtained by calculating the correlation between the 9-level index limited value and the reference sample, that is, the corresponding relationship between the correlation and the level. For any group of the index values, they need to be got the correlation associated with the reference sample, their level can be determined.

The steps for calculating the correlation:

1) The matrix between reference samples and measured values is determined as formula (17), and then need to normalize. 


$$
\mathbf{X}=\left[\begin{array}{cccc}
x_{01} & x_{02} & \cdots & x_{0 m} \\
x_{11} & x_{12} & \cdots & x_{1 m} \\
\vdots & & & \\
x_{n 1} & x_{n 2} & \cdots & x_{n m}
\end{array}\right]
$$

In the type: the first row is the reference sample, the rest is the comparison samples, $m$ stands for the amount of measured indices, $n$ stands for the amount of the comparison samples.

2) The absolute values of the difference are calculated in the first row and the rest, and make up the matrix as formula (18).

$$
\left[\begin{array}{cccc}
\Delta_{11} & \Delta_{12} & \cdots & \Delta_{1 m} \\
\Delta_{21} & \Delta_{22} & \cdots & \Delta_{2 m} \\
\vdots & & & \\
\Delta_{n 1} & \Delta_{n 2} & \cdots & \Delta_{n m}
\end{array}\right]_{n \times m}
$$

In the type: $\Delta_{i j}=\left|x_{0 j}-x_{i j}\right|(i=1,2, \cdots, n ; j=1,2, \cdots, m)$.

3) The data of the formula (18) transforms as the formula (19), and gets the matrix of the correlation coefficient as formula (20).

$$
\begin{aligned}
& \varepsilon_{i j}=\frac{\min _{i} \min _{j}\left\{\Delta_{i j}\right\}+\rho \max _{i} \max _{j}\left\{\Delta_{i j}\right\}}{\Delta_{i j}+\rho \max _{i} \max _{j}\left\{\Delta_{i j}\right\}}=\frac{\Delta_{\min }+\rho \Delta_{\max }}{\Delta_{i j}+\rho \Delta_{\max }} \\
& {\left[\begin{array}{cccc}
\varepsilon_{11} & \varepsilon_{12} & \cdots & \varepsilon_{1 m} \\
\varepsilon_{21} & \varepsilon_{22} & \cdots & \varepsilon_{2 m} \\
\vdots & & & \\
\varepsilon_{n 1} & \varepsilon_{n 2} & \cdots & \varepsilon_{n m}
\end{array}\right]_{n \times m}}
\end{aligned}
$$

In the type: $\rho$ is the resolution coefficient, and $\rho \subseteq(0,1)$, its value is most in the range of $0.1 \sim 0.5$ as formula (20) in general. The smaller the resolution coefficient $\rho$ is, the more the difference between the correlation coefficients can be improved. The principle of selected value $\rho$ [16]:

When the conditions $\Delta_{\max }>3 \bar{\Delta} \& \& \varepsilon_{\Delta} \leq \rho \leq 1.5 \varepsilon_{\Delta}$ are met, the value $\rho$ is $1.5 \varepsilon_{\Delta}$;

When the conditions $\Delta_{\max } \leq 3 \bar{\Delta} \& \& 1.5 \varepsilon_{\Delta} \leq \rho \leq 2 \varepsilon_{\Delta}$ are met, the value $\rho$ is $2 \varepsilon_{\Delta}$.

In the type: $\varepsilon_{\Delta}=\frac{\bar{\Delta}}{\Delta_{\min }}, \bar{\Delta}$ is the mean of all absolute difference, that is, $\bar{\Delta}=\frac{1}{n \cdot m} \sum_{i=1}^{n} \sum_{j=1}^{m} \Delta_{i j}$.

4) The correlation between the reference samples and the comparative ones is calculated as formula (21).

$$
r_{i}=\frac{1}{m} \sum_{j=1}^{m} \omega_{i j} \varepsilon_{i j}
$$

In the type: $\omega_{i j}$ is the dynamic weight we obtain.

\section{Example analysis}

Based on the above method, the monitoring data of $110 \mathrm{kV}$ voltage level in $220 \mathrm{kV}$ Xindu substation and 110kV Huifeng substation of Guangxi is calculated and analyzed, the measured data is shown in Table 3.

Table 3 the measured data of power quality $(110 \mathrm{kV})$

\begin{tabular}{|c|c|c|c|c|c|}
\hline Substation & $\begin{array}{c}\text { Voltage Deviation } \\
X_{1} \%\end{array}$ & Frequency & \multicolumn{2}{|c|}{ Total Harmonic } & \multicolumn{2}{|c|}{ Long Time } & \multicolumn{2}{c|}{ Three Phase Unbalance } \\
Deviation $x_{2}$ & Distortion $x_{3} \%$ & Flicker $x_{4}$ & Degree $x_{5} \%$ \\
\hline Xindu & 6.397 & 0.059 & 5.465 & 0.77 & 2.038 \\
\hline Huifeng & 5.115 & 0.043 & 4.19 & 0.64 & 1.83 \\
\hline
\end{tabular}


The steps for the analysis:

1) The order relation of the index $x_{1} \succ x_{2} \succ x_{3} \succ x_{4} \succ x_{5}$ and the comparison vector $\mathbf{r}=[1,1.2,1.4$, $1]$ are determined, the subjective weight $\omega=[0.2485,0.2485,0.2071,0.1479,0.1479]$ is obtained by G1 method.

2) All the measured values consist of a matrix, and the matrix is weighting process by using the subjective weight, the comprehensive weight $\omega=[0.2745,0.2637,0.1349,0.1640,0.1629]$ is obtained by the SD method.

3) The comprehensive weight is adjusted by the cask effect, and the dynamic weight we obtain $\boldsymbol{\omega}=[0.1856,0.0822,0.3897,0.1669,0.1755 ; 0.1851,0.0730,0.3725,0.1730,0.1965]$.

4) The standard sample we make the rule is $X_{0}=[0,0,0,0,0]$. Each level boundary of the index and two substations' measured values make the correlation with standard sample by the GRA method, as shown in Table 4.

Table 4 the level boundary of the correlation

\begin{tabular}{|c|c|c|c|c|c|}
\hline level & 1 & 2 & 3 & 4 & 5 \\
\hline correlation & $\geq 0.8925$ & $\geq 0.8195$ & $\geq 0.7667$ & $\geq 0.7255$ & $\geq 0.6917$ \\
\hline level & 6 & 7 & 8 & 9 & - \\
\hline correlation & $\geq 0.6382$ & $\geq 0.5967$ & $\geq 0.5630$ & $<0.5630$ & - \\
\hline
\end{tabular}

Compared with another methods [8][9][15], their evaluation results of power quality in two substations are shown in Table 5.

Table 5 the comparison of the evaluation results

\begin{tabular}{|c|c|c|c|c|c|}
\hline Substation & correlation & Result of this paper & Result of the paper [8] & Result of the paper [9] & Result of the paper [15] \\
\hline Xindu & 0.6058 & 7 & 5 & 6.627 & 9 \\
\hline Huifeng & 0.6519 & 6 & 4 & 4.902 & 8 \\
\hline
\end{tabular}

As far as we know from the measured values in two substations, the total harmonic distortion of two substations is more than two times than the standard value, and belongs to the serious situation, the Xindu is in the ninth level and the Huifeng is in eighth level. The three phase unbalance degree of the Xindu also exceeds the standard value, and stays in the sixth level. Therefore, comprehensive power quality level should be unqualified, but the paper [8] shows the result that the level of the Xindu is good and one of the Huifeng is qualified, the paper [9] provides a result that the level of the Huifeng is qualified, it is obviously unreasonable. The results of the paper [15] are too extreme, without considering the weight of different indices. In this paper, the evaluation results are that the Xindu is moderate pollution and the Huifeng is light pollution. Compared with other methods, the conclusion of this paper is the most reasonable.

\section{Conclusion}

In this paper, the comprehensive evaluation method of power quality is proposed with considering the sensitivity of different power quality indices on the loads. The weight we obtain is a comprehensive of the subjective weight and the objective one, it will change with the measured values of the index. The example shows that the method we proposed in this paper is good practical and reasonable.

\section{Acknowledgement}

In this paper, the research was sponsored by Guangxi Power Grid of China Southern Power Grid (Project Name. Design and application of the monitoring equipment calibration system based the standard source, No. K-GX2014-023). 


\section{References}

[1] Xiangning Xiao. Analysis and Control of Power Quality [M]. Beijing: China Electric Power Press, 2004.

[2] Xiaodong Yuan. Multi-level Fuzzy Comprehensive Evaluation of Power Quality[C]. 2004 IEEE International Conference on Electric Utility Deregulation, Restructuring and Power Technologies April 2004 Hong Kong, 2004:290-294.

[3] Sidong Xian. Fuzzy Comprehensive Evaluation Model based on SVM[C].Second International Symposium on Intelligent Information Technology and Security Informatics,2008:97-100.

[4] Xia Zhao, Chengyong Zhao, Xiufang Jia, et al. Fuzzy Synthetic Evaluation of Power Quality Based on Changeable Weight[J].Power System Technology, 2005, 29(6):11-16.

[5] Rui Wang, Jie Fang, Ke Zhang, et al. Fuzzy Synthetic Evaluation of Power Quality Based on Entropy and AHP[J]. Electrical Measurement and Instrumentation, 2007,44(503):21-25.

[6] Shuai Yuan, Weiming Tong. A Novel Method for Power Quality Comprehensive Evaluation Based on ANN and Subordinate Degree[C]. Fourth International Conference on Natural Computation,2008:62-65.

[7] Yingying Liu, Guodong Li, Qiang Gu, et al. The Comprehensive Evaluation of Power Quality Based on the RBF Neual Network [J]. Electrical Applications, 2007,26(1):45-48.

[8] Xiufang Jia, Weiqing Ma. Quantified Assessment of Load Disturbing Property [J]. Journal of North China Electric Power University, 2008,35(4):7-12.

[9] Yingying Liu, Yonghai Xu, Xiangning Xiao. Analysis of New Method on Power Quality Comprehensive Evaluation for Regional Grid[J]. Proceedings of the CSEE, 2008, 28(22): 130-136.

[10] Yan Jin, Yingying Liu, Yonghai Xu. Analyse and Research on the Comprehensive Evaluation of the Power Quality [J]. Electrical Engineering, 2006(1):27-30.

[11] Lianjie Li, Jiangang Yao, Libo Long, et al. Application of Combination Weighting Method in Fuzzy Synthetic Evaluation of Power Quality [J]. Automation of Electric Power Systems, 2007, 31(4):56-60.

[12] Yajun Guo. The theory, method and application of comprehensive evaluation[M]. Beijing: Science Press, 2007.

[13] Xuejun Wang, Yajun Guo. Analyzing the Consistency of Comparison Matrix Based on G1 Method[J]. Chinese Journal of Management Science, 2006, 14(3): 65-70.

[14] Yicheng Ye, Huali Ke, Deyu Huang. Comprehensive Evaluation System and its Application[M]. Beijing: Metallurgical Industry Press, 2006.

[15] Shun Tao, Xiangning Xiao. Synthetic grading evaluation of power quality based on incentive mechanism [J]. Advanced Technology of Electrical Engineering and Energy, 2008, 27(2): 16-20.

[16] Feng Lu. Research on the Identification Coefficient of Relational Grade for Grey System [J]. Systems Engineering theory \& Practice, 1997 (6):49-54. 